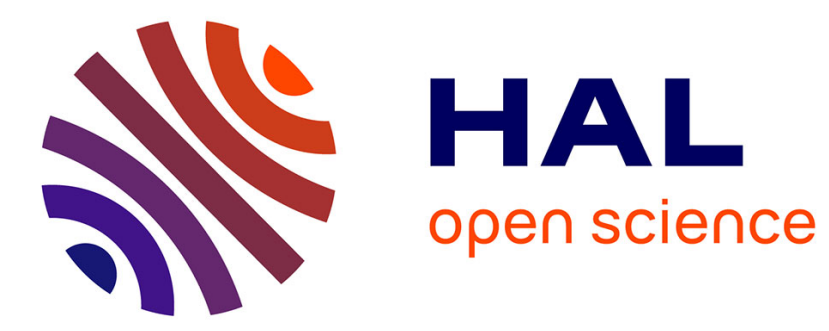

\title{
Infrared system for 3D scanning of metallic surfaces
}

\author{
Olivier Aubreton, Alban Bajard, Benjamin Verney, Frederic Truchetet
}

\section{To cite this version:}

Olivier Aubreton, Alban Bajard, Benjamin Verney, Frederic Truchetet. Infrared system for 3D scanning of metallic surfaces. Machine Vision and Applications, 2013, 24, pp.1513-1524. 10.1007/s00138013-0487-z . hal-00864695

\section{HAL Id: hal-00864695 \\ https://hal.science/hal-00864695}

Submitted on 23 Sep 2013

HAL is a multi-disciplinary open access archive for the deposit and dissemination of scientific research documents, whether they are published or not. The documents may come from teaching and research institutions in France or abroad, or from public or private research centers.
L'archive ouverte pluridisciplinaire HAL, est destinée au dépôt et à la diffusion de documents scientifiques de niveau recherche, publiés ou non, émanant des établissements d'enseignement et de recherche français ou étrangers, des laboratoires publics ou privés. 


\title{
Infrared system for 3D scanning of metallic surfaces
}

\author{
,O.Aubreton, A.Bajard, B.Verney, F.Truchetet \\ Laboratoire Le2i, UMR CNRS 6306, 12 rue de la fonderie, 71200 Le Creusot, \\ France \\ Phone: +33 (0)3 857310 00, Fax: +33 (0)3 857310 99, \\ olivier.aubreton@u-bourgogne.fr alban.bajard@u- \\ bourgogne.fr ; benjamin.verney@welience.com ; frederic.truchetet@u-bourgogne.fr
}

\begin{abstract}
Many experimental techniques and many commercial solutions have been proposed in order to realized non contact 3D digitization of industrial objects. Unfortunately, the performances of active 3D scanners depend on the optical properties of the surface to digitize. That is why, the results obtained by active $3 \mathrm{D}$ triangulation on specular or transparent surfaces are not as good as these obtained on diffuse surfaces. In this paper we present the developments we have realized, to address highly reflective metallic surfaces. These developments are based on the extension of a technique, called "Scanning From Heating" and initially dedicated to glass material. In comparison to conventional active triangulation techniques that measure the reflection of visible radiation, we measure here the thermal emission of a surface, which is locally heated by a laser source. We describe in this paper the successive steps we have followed in order to adapt Scanning From Heating to metallic materials, to evaluate the performances and finally to develop an operational prototype.
\end{abstract}

Keywords : 3D Scanning, Infrared, Scanning From Heating, Specular surfaces, Prototype

\section{3D digitization of specular surfaces}

3D reconstruction of surfaces is an important topic in computer vision and corresponds to a large field of applications: industrial inspection, reverse engineering, object recognition, biometry, archeology... Because of the large varieties of applications, we can find in the literature a lot of approaches whose can be classified into two families: passive and active [1]. Certainly because of their reliability, active approaches, using imaging system with an additional controlled light source, seem to be the most commonly used in the industrial field. In this domain, the 3D digitization approach based on active 3D triangulation has had important developments during the last ten years [2] and seems to be mature 
today if we consider the important number of systems proposed by manufacturers. Unfortunately, the performances of active 3D scanners depend on the optical properties of the surface to digitize. As an example, on Fig 1.a, a 3D shape with a diffuse surface has been digitized with Comet V scanner (Steinbichler). The 3D reconstruction is presented on Fig 1.b. The same experiment has been done on the same shape presenting a specular surface (Fig 1.c and Fig 1.d) where we can observe the influence of specularity on the performance of the digitization.

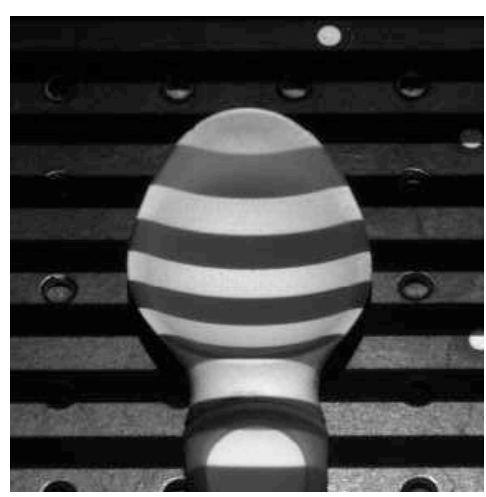

a)

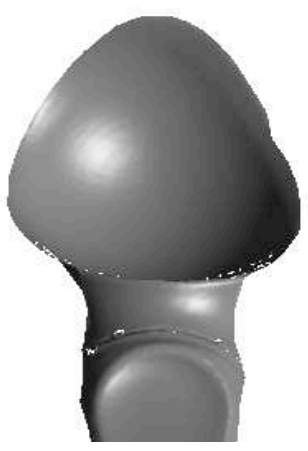

b)

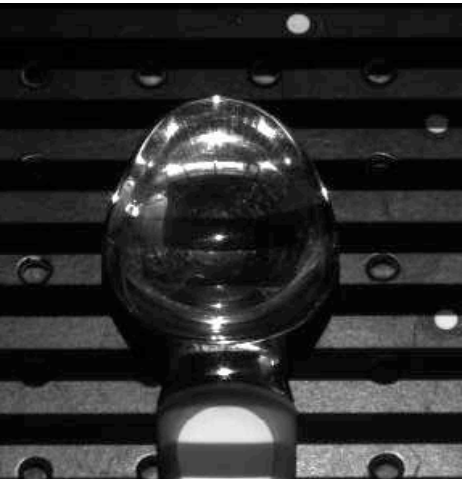

c)

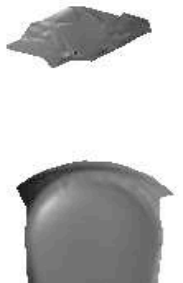

d)

Figure 1 : Distortion of the structured light pattern and the corresponding 3D digitization results for a powdered surface and steel with a mirroring surface

A lot of approaches have been proposed in the past in order to solve the problem of digitization of non-cooperative surfaces (specular surfaces, but also glossy, transparent...). In [3] Irkhe et al present a consequent state of the art and a classification of the experimental 3D digitization solutions according to the optical particularities of the surface. Among the cited solutions, Park and Kak [4] object to the basis assumption of an active scanner, considering that a projected point corresponds to a single point in the image frame, and they propose to measure all the points that are reflected by the specular object toward the sensor 
(resulting of inter-reflections). Then, the algorithm iteratively removes false points through a series of tests. Because several images have been acquired from different points of view, constraints can be applied to these tests, but the thresholds may need to be defined a priori. Although the results allow for a complete visualization of the glossy object, the accuracy is not sufficiently good for measurement applications or for surface inspection.

Approaches based on "Shape from Polarization" have also been the topic of many research studies on specular surfaces (and transparent surfaces). Wolff [5] proved the relation between the surface normal and the polarization parameters of the reflected ray at the surface of the material. Then, the technique was extended to $3 \mathrm{D}$ digitization of specular metallic objects [6], especially by means of active lighting [7]. Even if the measurement accuracy is good, this method requires a confined environment, which limits the size of the objects that can be reconstructed.

While in the visible range, specular reflections are usually considered to be noise in the reconstruction algorithms, deflectometry take advantage of specular reflections to compute the depth and orientation of the surface. Tarini et al [8] propose an approach called "Shape from Distortion". They proposed to observe the distortion of several light patterns that were projected from a diffuse source (a computer monitor) on the mirroring surface. Bonfort et al [9] propose a similar method and show that it is possible to recover the shape of specular surfaces by projecting a light pattern from two unknown positions. For these techniques, the cloud of points that is obtained could be very dense, but the matching constraint is high, and a high curvature shape cannot be fully recovered from one pose.

Another method, sometimes classified in the "Shape from Specularities" category, was developed by Zheng [10] and has been widely used. The principle relies on the tracking of a specular reflection that is induced by light sources surrounding the object, when the object is rotating. The continuous acquisition by a fixed camera provides a spatiotemporal collection of images. The study of the apparent motion of specular highlights on the object will help to recover the whole surface. However, the authors noted that the reconstruction accuracy depends strongly on the nature of the specular surface. We note that this remark highlights a general drawback to all of the non-conventional techniques that were mentioned above: the presence of any diffuse part on the object will interfere with 
the points cloud digitization. In other words, the diffuse surfaces cannot be entirely reconstructed by this type of technique.

Other approaches such as multi-camera passive systems [11], structuredlight based systems [12], have also been proposed, but the surface roughness should not exceed a low bound. "Shape from Fluorescence" technique [13] is a recent promising approach. The authors use an UV laser in order to generate a visible pattern on the surface, using fluorescence effect. The accuracy is similar to the one obtained using conventional scanners on a diffuse surface. But the main limitation is that the fluorescence in visible domain doesn't exist on all the material. In other words, the method is not applicable on all the metals (steel for example). Chromatic confocal microscopy [14] is another way to estimate the $3 \mathrm{D}$ coordinates of an opaque surface with good accuracy, but the surface should not be excessively tilted. Finally we can note that some commercial systems mounted on robotic arms (Nikon Metrology) operate on the assumption that the reflected radiation at the surface of an object has a quite diffuse component; thus, scanners can acquire a reflective shape after several successive scans, if the incident radiation is sufficiently intense and if the distance of the measurement is relatively low.

We can note that the approaches presented previously, exploit specularity of the surface in order to realize $3 \mathrm{D}$ reconstruction. That is why each of them is generally not adapted on surfaces whose present different optical properties. A second remark is that all these approaches work in the visible domain and non explore other spectral domains, such as for example, infrared domain.

In this paper, we will present some developments based on using infrared imaging in order to realize an operational 3D scanner able to digitize metallic objects with low dependence to the optical property of the surface. The method we studied, called "Scanning from Heating", has been initially proposed to realize 3D scanning of transparent glass. The evolution to digitization of metallic objects will be presented in section 2, with the evaluation of the performances obtained on a first experimental setup. In section 3 we will present an evolution of this setup, in order to realize an operational 3D scanner. After having the system described, the scanning results will be presented and commented. Finally we will conclude in section 4 and present some future works. 


\section{Scanning from heating: application to metallic objects}

\section{a. Infrared imaging and $3 d$ digitization}

Infrared imaging is well known to be used to realize non-contact temperature measurement, defect detection, non-destructive control, and so on. But researchers have been also interested in designing 3D acquisition systems in infrared domain, for example to realize invisible structured light 3D scanners. Kinect (C) Microsoft is certainly the most popular one. Other example can be found in [15] where authors propose to use infrared structured light imaging for $3 \mathrm{D}$ airbag generation. An other way has been presented in [16] where the authors describe an omnidirectional sensor using infrared structured light. More recently, authors present in [17] an infrared stereoscopic system dedicated to combine temperature measurement, 3D shape and displacement of a heated metallic object. As the authors explain, the matching between the two images is not so easy because of the low contrast in the thermal images. IR polarization imaging system has been experimented and presented in [18]. The choice of the camera (long wave infrared camera) permits to obtain images in a spectral domain where the glass is opaque. The 3D reconstruction is obtained by analyzing the parameters of polarization of the IR light reflected on the surface. These techniques correspond to an application in infrared domain of methods developed in the visible domain (see section 1), based on the analyzing of reflected light on the surface and whose present similar limitations to optical properties of the object.

A second way, of 3D digitization in IR domain, seems to have been inspired by the active thermography, commonly used in non-destructive testing applications. In [19] Bodnar presents a flying spot laser system, which is able to detect and evaluate the dimensional characterization of wear, cracks that occur in metallic materials. The method used is called "photothermal radiometry", and is based on scanning the object with a laser excitation. In the same field, Pelletier and Maldague [20] have developed a technique for thermal image analysis called "Shape from Heating", which can extract the orientation and depth of simple surface (a cylinder in the paper) that is heated by a diffuse infrared source. The main goal of this 3D shape extraction is to improve the detection of defects by correcting the temperature variations that result from the non-planarity of the surface. Even though the accuracy of this method has not been demonstrated yet 
for the 3D digitization of complex shapes, this study was among the first to consider thermography to extract 3D information.

\section{b. Scanning From Heating}

The studies presented in this article on the 3D scanning of specular surfaces are related to a non-conventional infrared approach introduced by Eren [21] for the 3D digitization of glass objects. Unlike classical active triangulation approaches, the principle of this technique, called "Scanning From Heating" ( $\mathrm{SFH})$, is based on the measurement of the infrared radiation that is emitted by the object instead of the reflection of visible radiation. A laser source is used to cause a local elevation of temperature; the 3D coordinates of each point are then extracted from the IR images with a prior geometric calibration of the system. As the emissivity is omnidirectional for most materials, the model of infrared radiation tends to approach the model of the diffuse reflection of visible light for diffuse surfaces and, thus, addresses the issue of specular reflection or transparency in the visible spectrum. Using a $\mathrm{CO}_{2}$ laser, the feasibility of this concept has been demonstrated on glass objects, and some scanning results have been presented [22]. The evolution of this work is the study of the application of scanning from heating to perfom 3D scanning of metals, specular or not, that have a high reflectivity and a high thermal conductivity.

\section{c. Application to highly conductive materials}

In a heat transfer problem, we need to evaluate the energy balance for the three exchange modes that are: conduction, convection and radiation. In the case of "Scanning From Heating" (see Fig. 2), the heat quantity is brought by a laser and has to be adjusted according to the thermophysical properties of the studied material and the thermal sensitivity of the camera. The generated heated point has to be intense enough to be detected by the sensor but it should not exceed a temperature limit, which would damage the surface by melting or marking. 


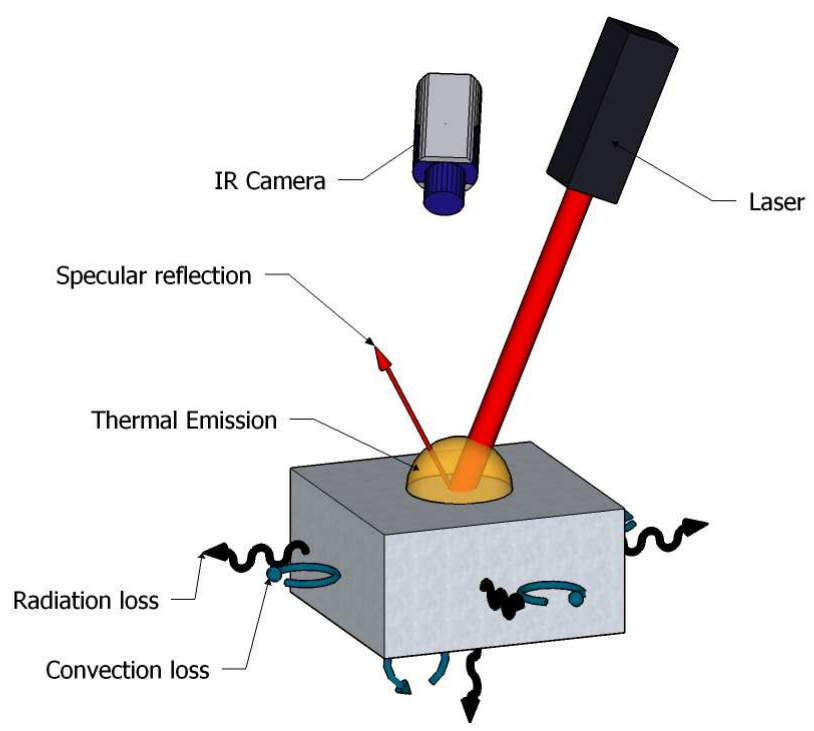

Figure 2 : Heat transfers during Scanning From Heating

Given that convection and radiation are only surface exchanges, we can consider two different elements of the material to write the global equation: the elements on the surface of the material and the elements within the volume. For any volume element $d V$ of the material surface, the heat stored causes a temperature rise [23] as follows:

$$
d Q_{t o t}=\rho d V C_{p} \frac{\partial \theta}{\partial t} d t
$$

where $d Q_{t o t}$ is the total quantity of heat accumulated in the element $d V, \rho$ is the density of the material and $C_{p}$ is its specific heat capacity and $\theta$ the temperature. Then, we can demonstrate that the sum of heat transfer components gives:

$$
\rho C_{p} \frac{\partial \theta}{\partial t} d t=k\left(\frac{\partial^{2} \theta}{\partial x^{2}}+\frac{\partial^{2} \theta}{\partial y^{2}}\right) d z-k \frac{\partial \theta}{\partial z}-\varepsilon \sigma \theta^{4}-h_{a i r}\left(\theta-\theta_{a i r}\right)+\varphi_{a b s}
$$

The second member of the equation is respectively the sum of:

- the conduction term where $k$ is the thermal conductivity of the studied material

- the radiation term where $\varepsilon$ is the total emissivity of the surface

- the convection term where $\theta_{\text {air }}$ is the ambient temperature and $h_{\text {air }}$ is the heat transfer coefficient of the air during natural convection

- and the heat flux absorbed by the surface $d S$ from the laser beam is a Gaussian energetic distribution given by [24]: 


$$
\varphi_{a b s}=\alpha \frac{2 P_{i n}}{\pi r_{0}^{2}} e^{-2\left(\frac{x^{2}+y^{2}}{r_{0}^{2}}\right)}
$$

where $\alpha$ is the surface absorptivity for the laser wavelength, $P_{\text {in }}$ is the incident power of the laser and $r_{0}$ is the radius of the laser beam at the focal plane.

Concerning the elements within the volume (under the surface), the conduction transfer becomes uniform according to the three directions and equation (2) becomes the classical heat equation:

$$
\rho C_{p} \frac{\partial \theta}{\partial t}=k \nabla^{2} \theta
$$

By using a finite element numerical solver, we have shown that it is possible to solve this equation in any case of three-dimensional problem [25] Assuming that we know or estimate the thermophysical properties of the material, we can predict the best settings for the heating process (incident power, pulse time), to improve the efficiency of the digitization method. As an example in table 1, the laser power is computed for different pulse durations such that the temperature of the surface is increasing by $3 \mathrm{~K}$ from the initial temperature. $3 \mathrm{~K}$ is a detectability threshold depending on the intrinsic properties of the thermal sensor. Different common materials are used for the simulation and the diameter of the laser beam $(0.5 \mathrm{~mm})$ is chosen according to the spatial resolution of the camera.

\begin{tabular}{|c|c|c|c|c|c|c|c|}
\hline & $\mathbf{k}(\mathbf{W} / \mathbf{m} \cdot \mathbf{K})$ & $\boldsymbol{\rho}\left(\mathbf{k g} / \mathbf{m}^{\mathbf{3}}\right)$ & $\mathbf{C}_{\mathbf{p}}(\mathbf{J} / \mathbf{k g} \cdot \mathbf{K})$ & $\boldsymbol{\alpha}$ & $\mathbf{P}_{\mathrm{t}=20 \mathrm{~ms}}(\mathbf{W})$ & $\mathbf{P}_{\mathrm{t}=10 \mathrm{~ms}}(\mathbf{W})$ & $\mathbf{P}_{\mathrm{t}=1 \mathrm{~ms}}(\mathbf{W})$ \\
\hline Oxidized steel & 44,5 & 7850 & 475 & 0,3 & $\mathbf{1 , 6}$ & $\mathbf{1 , 8}$ & $\mathbf{4 , 1}$ \\
\hline Polished steel & 44,5 & 7850 & 475 & 0,07 & $\mathbf{7}$ & $\mathbf{7 , 8}$ & $\mathbf{1 7 , 5}$ \\
\hline Oxidized aluminum & 160 & 2700 & 900 & 0,25 & $\mathbf{5 , 9}$ & $\mathbf{6 , 2}$ & $\mathbf{9 , 1}$ \\
\hline Polished aluminum & 160 & 2700 & 900 & 0,05 & $\mathbf{2 9}$ & $\mathbf{3 1}$ & $\mathbf{4 4 , 7}$ \\
\hline Oxidized Copper & 400 & 8700 & 385 & 0,2 & $\mathbf{1 8}$ & $\mathbf{1 8 , 4}$ & $\mathbf{2 4 , 5}$ \\
\hline Tungsten & 174 & 19300 & 130 & 0,4 & $\mathbf{3 , 9}$ & $\mathbf{4 , 1}$ & $\mathbf{6 , 1}$ \\
\hline
\end{tabular}

Table 1 : Powers predicted to reach $3 \mathrm{~K}$ after $\mathrm{t}=1 \mathrm{~ms}, \mathrm{t}=10 \mathrm{~ms}, \mathrm{t}=20 \mathrm{~ms}$

\section{d. First Experiments}

Relying on the simulation results, a first experimental setup has been implemented, based on the SFH technique. . Concerning the choice of the incident wavelength, we can rely on the numerous databases of optical constants in the literature ([26], [27]). Indeed, the absorption coefficient of metallic surfaces tends 
to increase when the wavelength decreases, that is why the laser wavelength should be chosen in the near infrared spectral band.

The system consists of a diode laser emitting at $\lambda=808 \mathrm{~nm}$, and an infrared imaging detector, which is sensitive to mid-wavelength infrared (InSb quantumbased sensor for which the sensitivity spectral band extends from 1.5 to $5 \mu \mathrm{m}$ ). The system is fixed on a 3-axis moving table, and the studied object is motionless, to have a large scanning window (see Fig 3). The working distance between the camera and the object is $60 \mathrm{~cm}$ and the focal length of the laser optics is $15 \mathrm{~cm}$. A programmable controller is used to synchronize the laser emission, the camera trigger and the displacement of the table.

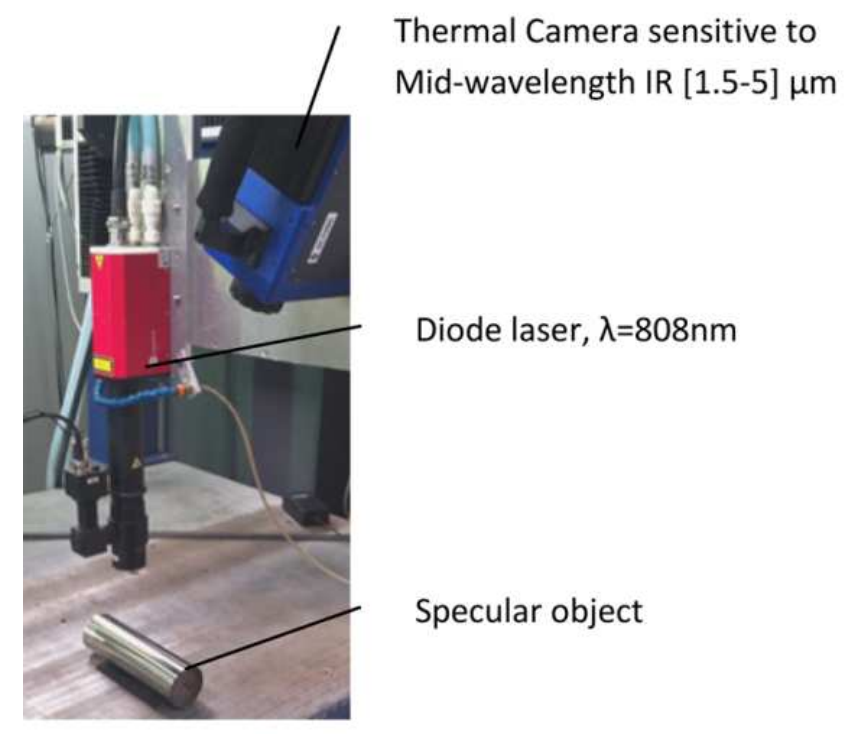

Figure 3 : Experimental setup

After setting the parameters of the laser source (pulse of $1 \mathrm{~ms}$ and power increasing from $4,1 \mathrm{~W}$ to $17,5 \mathrm{~W}$ - see table 1 ), the scanning procedure begins. For each intermediate position of the system, an image is acquired before and during the laser pulse, to facilitate the position computation of the thermal spot in the images, using background subtraction. A subpixellic method is used to obtain the maximum heat localization in the image frame. An initial calibration procedure has been realized before and gives the relationship between the coordinates of the spot in the image to the depth $\mathrm{Z}$ in the world coordinates and knowing the position of the moving platform the $3 \mathrm{D}$ coordinates of the measured point are determined.

Fig. 4 presents the 3D digitization results that were obtained for several specular objects with the system described above. 

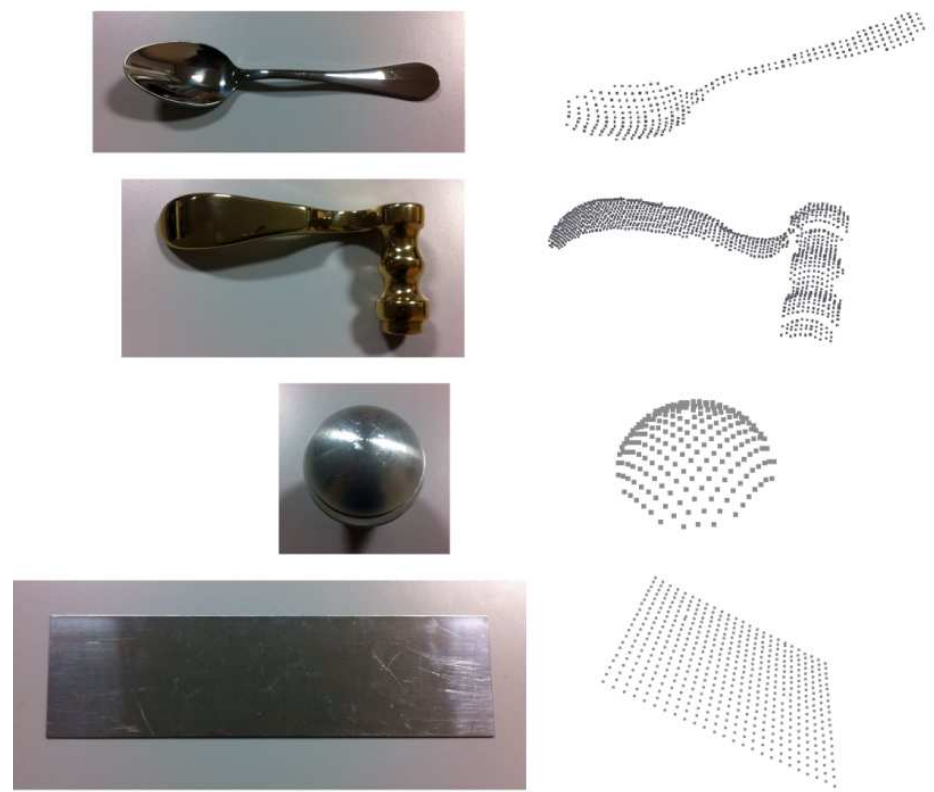

Figure 4 : Examples of 3D digitization results : polished steel, doorknob made of steel with a layer of brass, aluminum ball, steel plane

To efficiently compare the 3D scanning results for different surface conditions, we have used cylinders that were made of steel AISI 316L and that have identical dimensions but different average roughness's (see Fig. 5) obtained with various polishing techniques (mechanical or electrolytic). Fig. 6 presents an example of the deviation map between reference points obtained from the touchprobe scanner on a surface and the points that were digitized by conventional scanner (Fig. 6 a) and our experimental setup (Fig. 6 b).

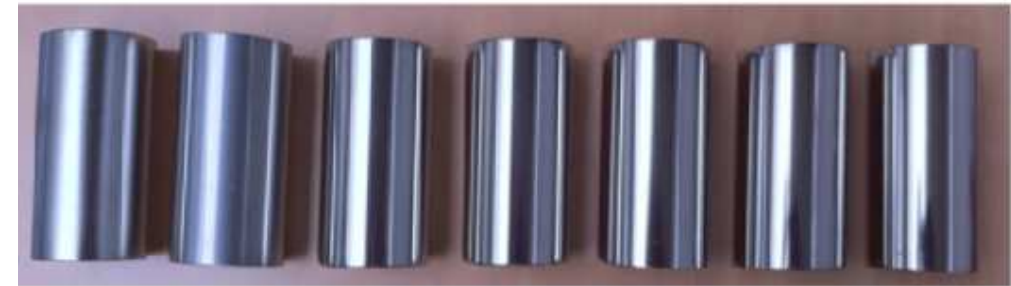

Figure 5 : Samples used for the evaluation of the accuracy of the Scanning From Heating 


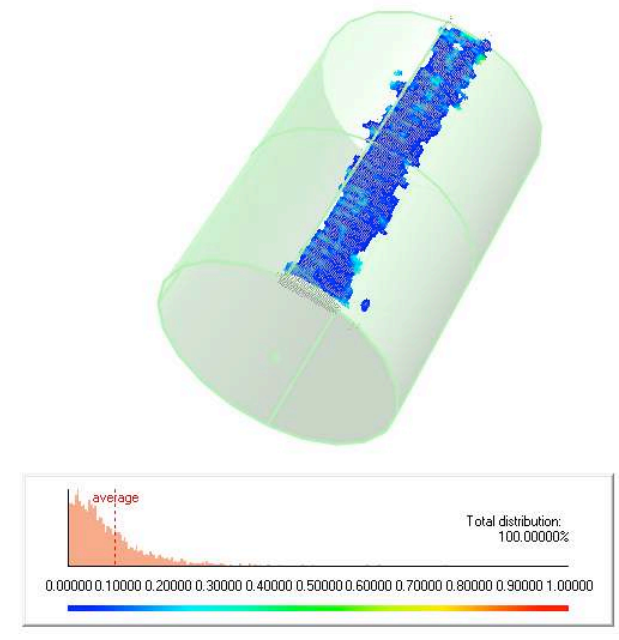

a)

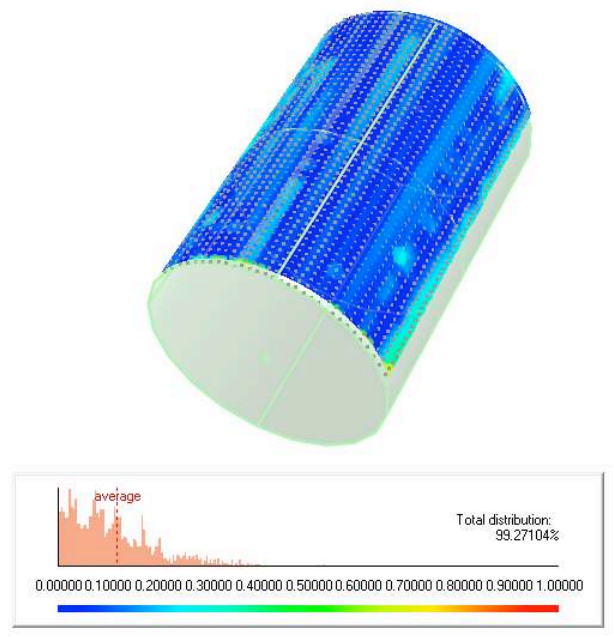

b)

Figure 6 : Deviation map and error distribution computed for the conventional scanner result (a) and for the SFH-based scanner result (b) and obtained for the most specular cylinder.

Concerning the eight samples, the overall average error is similar: $154 \mu \mathrm{m}$ with the SFH-based scanner and $174 \mu \mathrm{m}$ with the conventional scanner. But the standard deviation obtained with the Comet scanner is $\sigma=338 \mu \mathrm{m}$ (average for the eight measurements) and is larger than the one obtained with SFH $(\sigma=156 \mu \mathrm{m})$.

In the example presented in Fig. 6, the surface points for the SFH are acquired for an incidence angle of up to $\alpha_{\max }=62.2^{\circ}$, with a total average error of $117 \mu \mathrm{m}$. This value is quite similar for the eight cylinders and is not really dependent on the roughness. In comparison, for the traditional system, the maximum incidence angle is only $\alpha_{\max }=28.6^{\circ}$, with a total average error of 148 $\mu \mathrm{m}$. As the specularity of the surface increases, it can be noted that $\alpha_{\max }$ decreases to $6.2^{\circ}$.

Of course, the evaluated measurement accuracies we have summarized here (see [28] for a complete description of the evaluation process and results), depend on the quality of the fitting between point clouds obtained by the two scanners and the reference obtained with touch probe. They are also related to the cylindrical shape of the samples. A metrological study would be necessary to improve the evaluation of the global error of this prototype. However it appears that the quality of the points clouds obtained with this system is fair and that 
Scanning From Heating can give interesting results on metallic surfaces with low dependence to roughness.

But the system we used here to realize the experiments presents a great disadvantage. Because of the moving platform used here to realize the scanning process, the time we need to obtain one scan is consequent (about 3 seconds to obtain 1 point). A second point is that all the system can't be easily moved and this point limits the field of applications. That is why the development of a more compact and portable system has been done. It will be presented in the next section.

\section{Scanner prototype based on SFH}

\section{a. Hardware description}

According to the previous theoretical and experimental study, a SFH-based prototype has been developed with the aim of avoiding mechanical vibrations, increasing the accuracy of the scanning and speeding up the process. In place of the moving platform, we have chosen to use a galvanometer is order to scan the surface with the laser; it reduces significantly the dimensions of the system and improves the scanning speed and quality thanks to two mirrors.

The prototype can be seen in Fig. 7. The laser source is made of ytterbium fiber pumped by diodes, which delivers a $1.07 \mu \mathrm{m}$ laser beam and the power ranges from 0 to $50 \mathrm{~W}$.

The optical components are three lenses that are assembled through the laser optical path. The two first lenses constitute an afocal system reducing the beam diameter, the third one focuses the laser to a $0.44 \mathrm{~mm}$ beam waist at $500 \mathrm{~mm}$ with a $200 \mathrm{~mm}$ depth of field.

The galvanometer scan head is made up of two mirrors controlled by piezoelectric motors that can deflect the laser beam with $150 \mu$ rad step, in $\mathrm{X}$ and $\mathrm{Y}$ directions.

The infrared camera performs the thermal measurement with a bolometer detector, which is sensitive to Long-Wavelength $([8-13 \mu \mathrm{m}])$.

A workstation controls the synchronization between camera acquisition, laser emission, and galvanometer scanner, and then performs the $3 \mathrm{D}$ reconstruction from image analysis. 


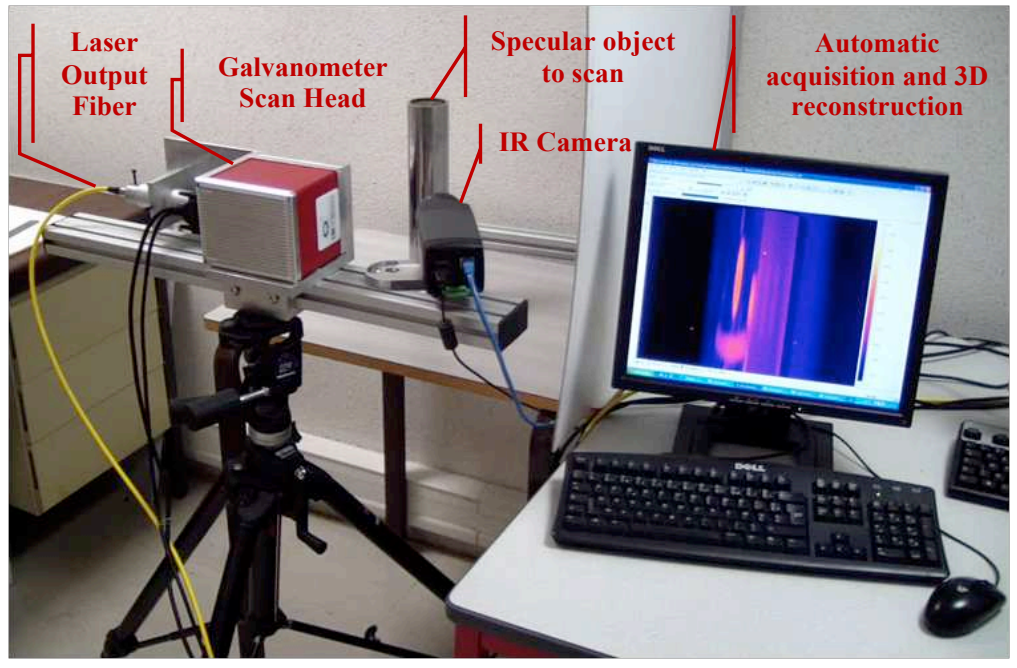

Figure 7: scanner prototype based on Scanning From Heating

\section{b. Calibration of the system}

Before the acquisition step, it is necessary to geometrically calibrate the laser-camera system. As the Rayleigh length of the laser (equivalent to the depth of field) is relatively long, we assume that there is no geometrical aberration, so the laser calibration is only based on the calculation of its extrinsic parameters, i.e. two rotations that are given by galvanometer mirrors. The electrical signals that command the mirror motors are linearly related to the deflection angles $\alpha_{x}$ and $\alpha_{y}$. The experimental determination of this relation is performed to know each position of the laser beam that is used for the scanning process.

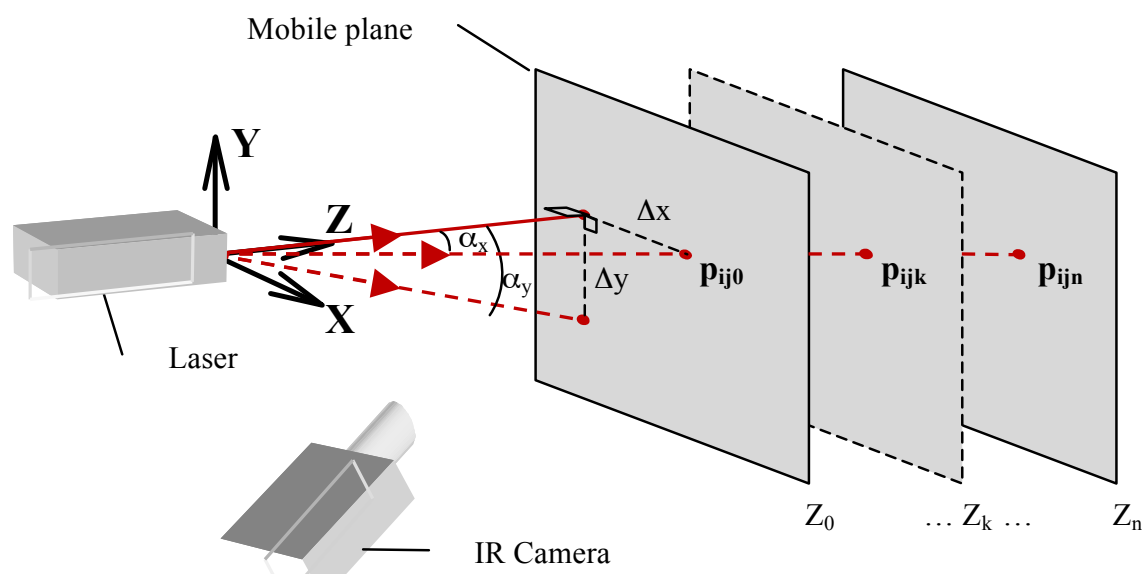

Figure 8 : Principle of the calibration process

The world frame is attached to the rotation center of the galvanometer scan head. After laser calibration step, we have to compute the transformation between 
the world frame and the image frame. The principle of the method we have implemented [29] is based on the rules of projective geometry. As illustrated in Fig. 8, a calibration plane is translated along $Z$ direction, perpendicularly to the laser incidence direction. Fig. 9 presents the sum of all the images obtained for 225 orientations of the laser beam, with an angle of $0.74^{\circ}$ between each of them, for one position of the plane.

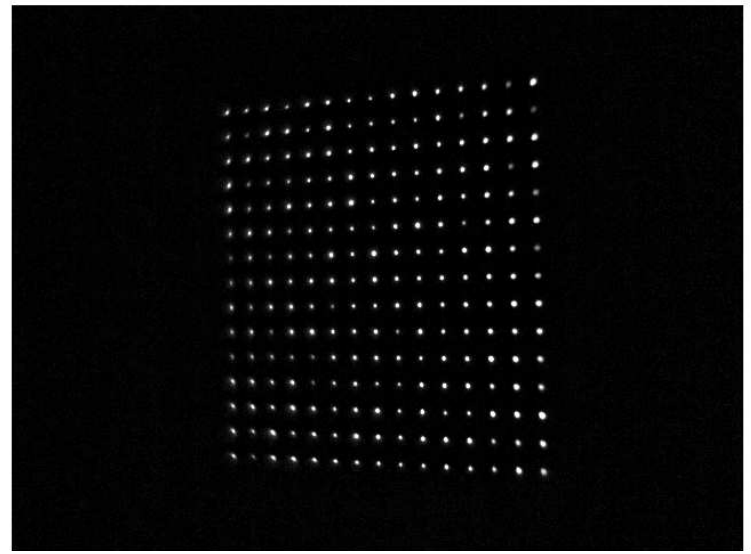

Figure 9 : Sum of all the IR images obtained for 225 orientations of the laser beam.

The plane is translated and $\mathrm{k}$ acquisitions are realized. Each point of the grid presented on Fig. 9, is moving along a line that corresponds to the projection of the laser beam in the image. An example can be seen in Fig.10 with k=3. Each color of points corresponds to one depth.

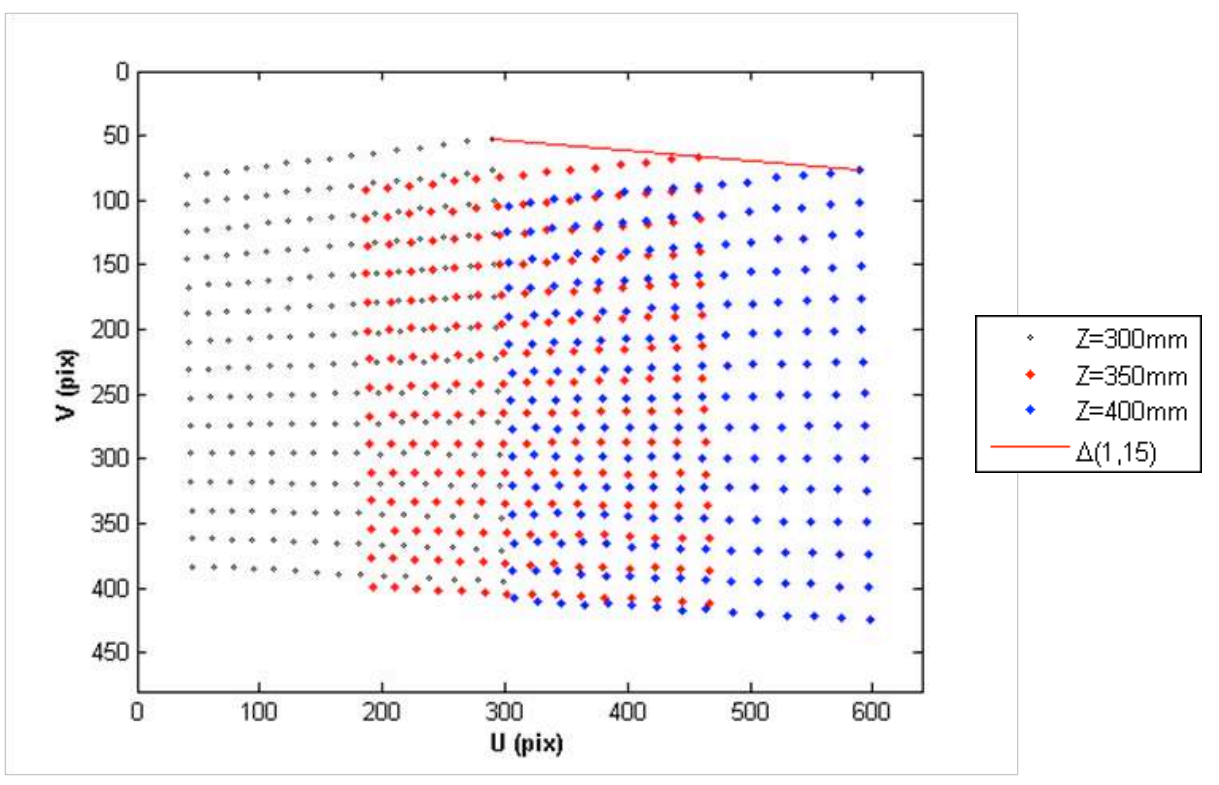

Figure 10 : Evolution of the point of the grid during the calibration process 
Using the $\mathrm{k}$ acquisitions, the equation of the line can be determine, and finally, for a $\mathrm{M}$ by $\mathrm{N}$ grid of points, $\mathrm{MxN}$ calibrations lines are determined :

$\Delta_{i j}: V_{i j}=a_{i j} U_{i j}+b_{i j}$ for $(i ; j) \in(M, N)$

For a position $k$ of the plane, we define $p_{i j k}$ as the $3 \mathrm{D}$ point corresponding to intersection of the laser, with an angle position of mirrors of the galvanometer $\left(\alpha_{i}, \alpha_{j}\right)$, and the plane and $p_{i j k}^{\prime}$, the corresponding point in the image. For each sampled position of the laser ray, five calibration parameters are computed. The first two parameters are the coefficients $a_{i j}$ and $b_{i j}$ of the equation of the straightline $\Delta_{i j}$ described by the points $p^{\prime}{ }_{i j k}$.

Then the relative position $D_{i j k}$ in pixels of every point is defined on each line $\Delta_{i j}$ :

$D_{i j k}=\left\|p_{i j k}^{\prime}-p^{\prime}{ }_{i j 0}\right\|$

with $p^{\prime}{ }_{i j 0}$ is the point obtained in the image for the initial position $Z_{0}$.

From projective geometry, it can be demonstrated that the relation between the coordinate $Z_{k}$ and the distance $D_{i j k}$ is given by the following:

$Z_{k}=\frac{c_{i j} D_{i j k}+d_{i j}}{e_{i j} D_{i j k}+1}$

where $c_{i j}, d_{i j}, e_{i j}$ are the three other parameters of the calibration.

An example of the relationship between $Z_{k}$ and the distance $D_{i j k}$ is presented in Fig. 11.

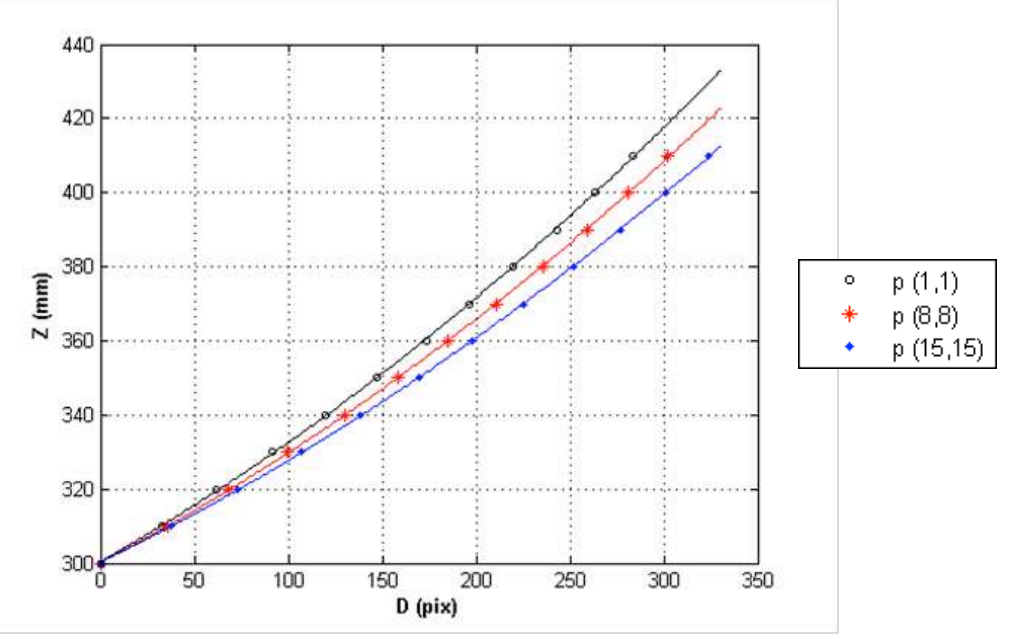

Figure 11: Examples of relationship between the depth $\mathrm{Z}$ and the relative position $\mathrm{D}$ of the hot spot. 
Knowing these five parameters and the angle position of the beam $\left(\alpha_{i}, \alpha_{j}\right)$, we can compute the three world coordinates of the considered point $p_{i j k}$ from the 2D coordinates of its corresponding point in the image frame.

\section{c. Results and discussions}

The galvanometer scan head seems to be an interesting choice in comparison to the moving table used in the first system. We obtain here a better speed of the scanning process: the point rate is now about 3 points per second and has been multiplied by 10 . In our experiments we have chosen to stop the laser between two measurements. So for each point the laser needs to be restarted, and this step requires about $250 \mathrm{~ms}$. Without this step, we have observed that some thermals patterns could be created on the material and observed by the camera, for example lines or grid patterns. The creation of 2D thermal patterns should increase the number of $3 \mathrm{D}$ points computed using only one infrared image and in extension the speed rate. But the quality of these patterns depends on the speed of the spot, on the power of the laser and on the physical properties of the material. A complementary study will be needed in order to experiment it.

The second difference is the IR camera. In the first experimental setup, the camera was a middle wave IR one [3-5] $\mu \mathrm{m}$. In the prototype, a long wave IR camera [8-13] $\mu \mathrm{m}$ has been used because of its theorical sensitivity to low temperature variation. The spatial resolution and the compactness of the camera (a Flir SC 655) was also a good point in our case.

The main limitation corresponds to some optical aberrations, which create errors on the detection of the hot spot in the IR images. An example can be seen on Fig.12. Fig 12.a) presents a zoom of the hot spot when it was in the middle of the image. Fig 12.b) corresponds to a zoom of the spot when he is near one border of the image. The distortion of the spot, observed in Fig. 12b). 5 hot spots have been created on a steel plate. They correspond to four points in the IR image in the four corners of the image and another one in center. We can note symmetry of the shape of the hot spots obtained in the corners with respect to the optical axis of the camera (i.e. the center of the image). We can also note that no distortion appears on the centered hot point. From this remark, we can guess that the geometrical aberration is related to the camera lens and we suppose that this distortion should be due to an optical distortion called "coma" effect, which can 
be met in the imaging systems with large spectral band (for example in the case of long wave IR cameras). This distortion appears when the incident rays are strongly inclined relative to the optical axis of the lenses of the camera and corresponds to optical distortions in the borders of the image.

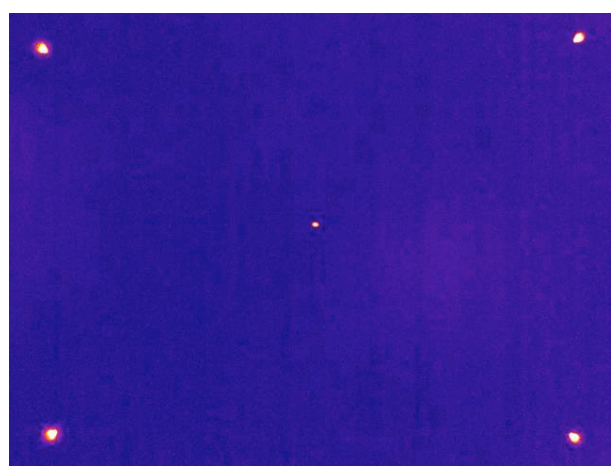

a) hot spots created on a steel plate
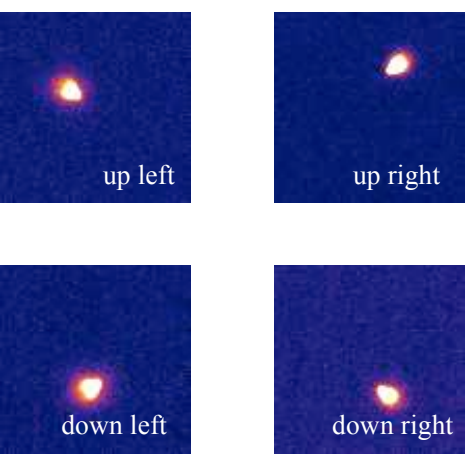

b) zoom of each hot spot

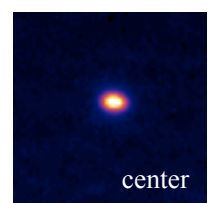

Figure 12 : Influence of the coma effect on the shape of the hot spot

This effect influences the localization of the hot spot when it is approaching the border of the image. An example of the deviation map obtained on a plane is presented on Fig. 13. The maximal deviations are localized on the border that seems to confirm the coma effect. The usual way to limit the coma effect is the use of optical filters for limiting the spectral domain of the camera. But this solution decreases the sensitivity of the camera and the contrast of the hot spot. Then it decreases the quality of the detection of the position of the spot in the image. The second solution, used here, is to take into account only the points which appear in an area, centered on the middle of the image, and where the distortion is considered as limited.
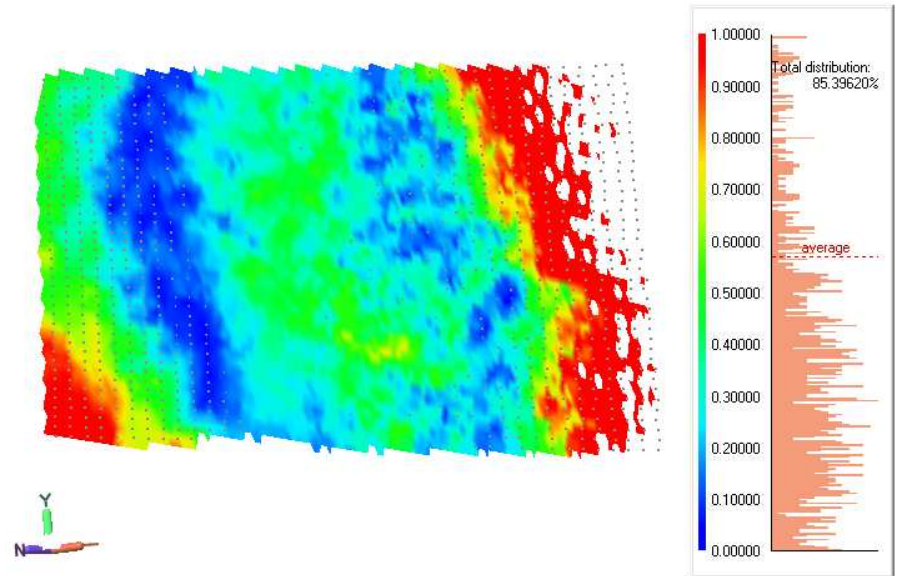

Figure 13 : influence of the distortion of the hot spot on the $3 \mathrm{D}$ digitization of a plane 
On Fig.14, some experiments on real objects are presented. They are obtained with the prototype described in section 3.a and using the calibration results previously seen. Experiments were conducted on different surfaces (specular or not) with various geometries with a resolution of 1600 points (40rows $\times 40$ columns). The laser beam orientations are regularly spaced by 0.3 deg. The value of the laser power has been chosen using simulation results summarized in table $1: 4,1 \mathrm{~W}$ for oxidized steel and 17,5 $\mathrm{W}$ for polished steel with a pulse time of $1 \mathrm{~ms}$. However, these values are only valid for materials having similar characteristics to those indicated in Table 1. Because the absorption coefficient of the objects presented in Fig.14 is less than the values used in simulations the incident laser power chosen for these examples is substantially more important (respectively 6W, 20W and 25W).

For each object we give the corresponding deviation map. Comparing to the previous experimental setup (where the vibrations of the moving table influences the accuracy of the measurement), the results are substantially better. As an example, the deviation map obtained with the specular cylinder, presents an average distance equal to $106 \mu \mathrm{m}$. For the same object, the error measured with the previous setup was about $206 \mu \mathrm{m}$. Using the 1600 points of the $3 \mathrm{D}$ point cloud, the cylinder diameter has been computed and presents an accuracy of 0.15 $\%$ comparing to the previous one which was about $0.41 \%$. Concerning the example of the spoon, the average error is $143 \mu \mathrm{m}$ and the standard deviation of the error distribution is $178 \mu \mathrm{m}$. The error is mainly localized on the edges, where the sampling step of the scanning is not fine enough.

In the experiments presented in this paper, we have observed that SFH can operate with similar accuracy on specular and non-specular surfaces (see the results obtained on the dark stairs) and the performance no longer depends on the roughness of the surface 

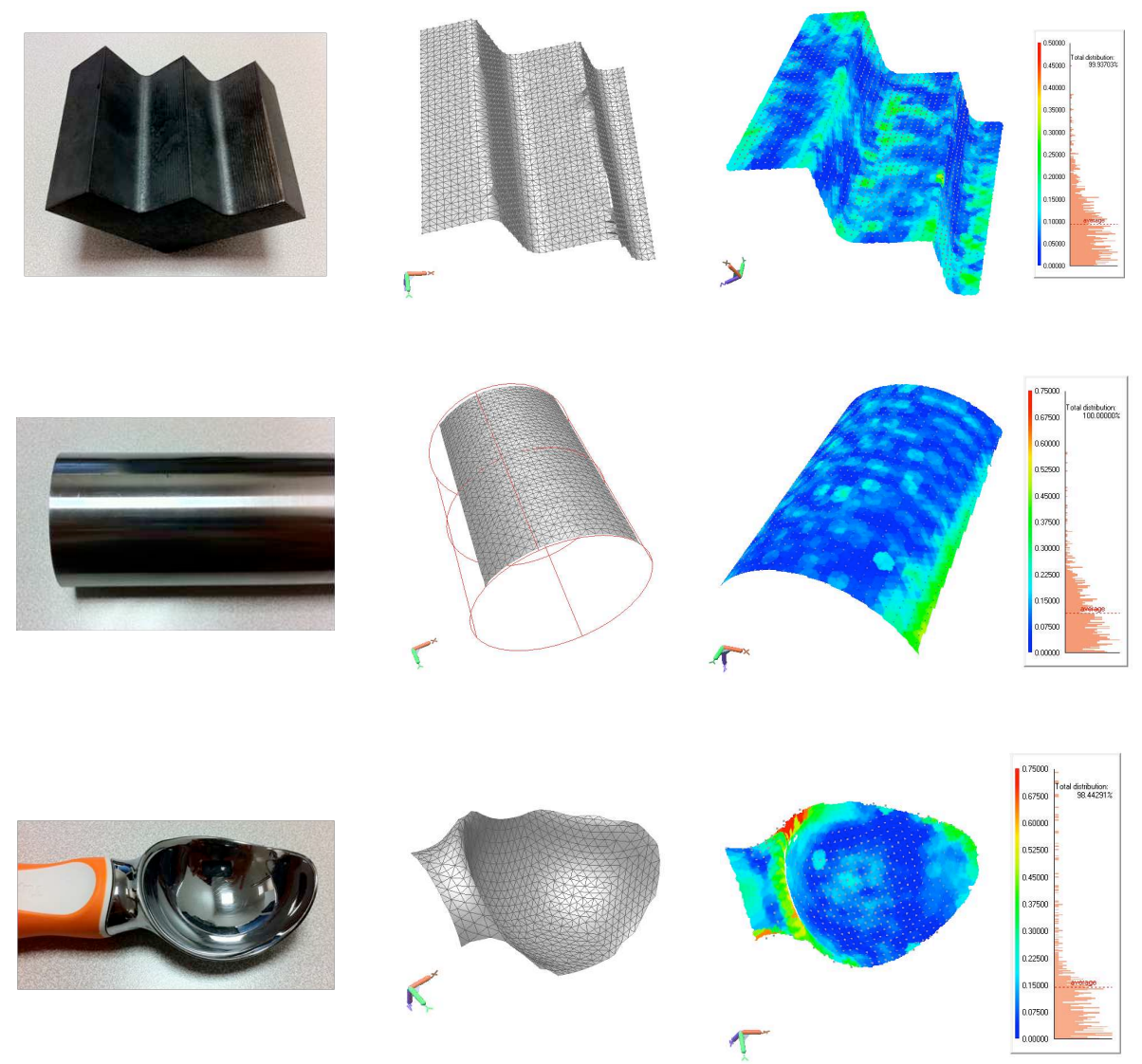

Figure $14: 3 \mathrm{D}$ digitization results obtained on : a) a non diffuse surface ; b) a secular cylinder ; c) a concave specular surface

\section{Conclusion}

This paper resumes the different steps followed to develop an operational 3D digitizer based on Scanning From Heating technic, and able to digitize metallic objects.

As the Scanning From Heating technique has been initially developed to realize $3 \mathrm{D}$ digitization of transparent glass, we have shown that a laser and an IR camera can be set up to digitize metallic specular surfaces in 3D. The study of thermophysical properties of metals, the modeling of the thermal problems, and the simulations permit to determine the characteristics of the laser (wavelength, power).

The performances of 3D reconstruction, obtained with a first experimental setup, have confirmed the choice of the laser and have also given a first evaluation of the accuracy. The average error of measurement is similar to the one obtained from a acquisition with conventional scanner, on diffuse surfaces. However, the standard deviation of the error distribution is lower and does not change 
significantly with the roughness, whereas for the conventional scanner, it increases with the specularity. Furthermore, the angle range of the acquired cloud of points is greater with the infrared system. In fact, with a similar error between the two systems and for the most specular surface, a 3D point can be computed with an incidence angle of up to $62^{\circ}$ with our system and only up to $6^{\circ}$ for the other system.

In order to improve the speed and the accuracy, a new prototype has been developed. A galvanometer head has been implemented in order to realize the scanning process and to avoid mechanical vibrations. The speed has been also improved and the accuracy obtained with this prototype is substantially better than the one obtained with the previous experimental system.

Comparing with others methods, we can note that the error measurement obtained with our Scanning From Heating system seems to be more important than the error presented with other approaches, for example approaches based on polarization imaging [7] or deflectometry [8], [9] (the maximal error obtained by these approaches on mirroring surfaces is less than $100 \mu \mathrm{m})$. But those techniques are dedicated to specular surfaces and don't seem to be applicable to other surfaces, for example on lambertian surfaces.

More generally speaking, a lot of approaches have been proposed in the past for 3D digitization of non-cooperatives surfaces (for example specular or transparent surfaces), but each one is generally linked to some optical properties of the surface in the visible spectrum. This point limits their field of application. In the experiments realized with Scanning From Heating approach, on specular and non-specular surfaces, the results obtained are similar to the ones obtained on diffuse surfaces with a conventional 3D scanner. So, Scanning From Heating is a 3D digitization technic using active triangulation technic, but it seems to be, in comparison with the systems developed in the visible domain, less dependent to the roughness of the surface (of course the absorption coefficient of the material, at the wavelength of the laser, must be superior to 0 ).

The main perspective is to generate $2 \mathrm{D}$ structured heated pattern on the surface in order to improve the speed of the scanning process. To do it, a compromise must be found between power and speed of the laser, knowing the themophysical properties of the material. This point will be studied in the future. 
As underline in [3] the problem of 3D digitization has been very studied by researchers in the past. 


\section{References}

[1] F. Blais, "Review of 20 years of range sensor development," Journal of Electronic Imaging, vol. 13, pp. 231-240, January 2004.

[2] G. Sansoni, M. Trebeschi, and F. Docchio, "State-of-The-Art and Applications of 3D Imaging Sensors in Industry, Cultural Heritagen Medicine, and Criminal Investigation," Sensors, vol. 9, pp. 568-601, 2009.

[3] I. Ihrke, K. N. Kutulakos, H. P. A. Lensch, M. Magnor, and W. Heidrich, "Transparent and Specular Object Reconstruction," Computer Graphics Forum, vol. 29, no. 8, pp. 1-27, December 2010 .

[4] J. Park and A.C. Kak, "3D Modeling of Optically Challenging Objects," IEEE Transactions on Visualization and Computer Graphics, vol. 14, pp. 246-262, 2008.

[5] L. B. Wolff and T. E. Boult, "Constraining object Features using a Polarization Reflectance Model," IEEE Transactions on Pattern Analysis and Machine Intelligence, vol. 13, no. 7, pp. 635657, 1991.

[6] S. Rahmann and N. Canterakis, "Reconstruction of Specular Surfaces Using Polarization Imaging," in IEEE Computer Vision and Pattern Recognition, Kauai, USA, 2001, pp. 149-155.

[7] O. Morel, C. Stolz, F. Meriaudeau, and P. Gorria, "Active Lighting Applied to 3D Reconstruction of Specular Metallic Surfaces by Polarization Imaging," Applied Optics, vol. 45, no. 17 , pp. 4062-4068, June 2006.

[8] M. Tarini, H. P.A. Lensch, M. Goesele, and H.-P. Seidel, "3D Acquisition of mirroring objects," Graphical Models, vol. 67, no. 4, pp. 233-259, 2005.

[9] T. Bonfort, P. Sturm, and P. Gargallo, "General Specular Surface Triangulation," in Asian Conference on Computer Vision, 2006, pp. 872-881.

[10] J. Y. Zheng and A. Murata, "Acquiring a complete 3D model from specular motion under the illumination of circular-shaped light sources," IEEE Transactions on Pattern Analysis and Machine Intelligence, vol. 22, no. 8, pp. 913-920, 2000.

[11] D. N. Bhat and S. K. Nayar, "Stereo and specular reflection," International Journal of Computer Vision, vol. 26, no. 2, pp. 91-106, 1998. 
[12] M. Gupta, A. Agrawal, and A. Veeraraghavan, "Structured light 3D scanning in the presence of global illumination," in IEEE Conference on Computer Vision and Pattern Recognition (CVPR), Providence, RI, 2011, pp. 713-720.

[13] R. Rantoson, "Numérisation 3D d'objets transparents par polarisation dans l'IR \& par triangulation dans l'UV," Université de Bourgogne, Thèse 2011.

[14] H. J. Tiziani and H. M. Uhde, "Three-dimensional image sensing by chromatic confocal microscopy," Applied optics, vol. 33, no. 10, pp. 1838-1843, Avril 1994.

[15] S. Boverie, M. Devy, F. Lerasle, "3D perception for new airbag generation", $15^{\text {th }}$ IFAC world Congress on Automatic Control, Barcelona, Spain 2002.

[16] O. Romain, T. Ea, C. Gastaud, P. Garda, "Un Capteur Multispectral de vision Panoramique 3D" Actes des Journées Francophones Des Chjercheurs En Ananlyse d'Images et Perception Visuelle (ORASIS'2001), Cahors, France, Juin 2001.

[17] J.-J. Orteu, Y. Rotrou, T. Sentenac, and L. Robert, "An Innovative Method for 3-D Shape, Strain and Temperature Full-Field Measurement Using a Single Type of Camera: Principle and Preliminary Results," Experimental Mechanics, vol. 48, no. 2, pp. 163-179, 2008.

[18] R. Rantonson, C. Stolz, D. Fofi, F. Meriaudeau, "Optimization of transparent objects digitization from visible fluorescence UV-induced", Optical Engineering, vol. 51, no 2, pp. 033601, April 2012

[19] J.L. Bodnar and M. Egée, "Wear Crack characterization by photothermal radiometry," WEAR, vol. 196, pp. 54-59, August 1996.

[20] J-F. Pelletier and X. Maldague, "Shape from heating: a two-dimensional approach for shape extraction in infrared images," Optical Engineering, vol. 36, no. 2, pp. 370-375, February 1997.

[21] G. Eren, O. Aubreton, F. Meriaudeau, L. A. Sanchez Secades, D. Fofi, A. T. Naskali, F. Truchetet, and A. Erçil, "Scanning From Heating : 3D shape estimation of transparent objects from local surface heating," Optics Express, vol. 17, no. 14, pp. 11457-11468, 2009.

[22] G. Eren, "3D Scanning of Transparent objects," Université de Bourgogne - Sabanc1 Üniversitesi, Thèse de doctorat, Septembre 2010.

[23] B. Eyglunent, Manuel de Thermique.: Hermès Science Publication, 2000

[24] R. . Paschotta. (2011) Gaussian Beams. Encyclopedia of Laser Physics and Technology. 
[25] A. Bajard, O. Aubreton, G. Eren, P. Sallamand, F. Truchetet, «3D Digitization of Metallic Surfaces Using Scanning From Heating Method Approach», SPIE Electronic Imaging San Francisco, 2011, Proc. SPIE 7864, 786413,

[26] Edward D. Palik, Handbook of Optical Constants of Solids. Boston: Academic Press, 1985.

[27] M. A. Ordal, L. L. Long, R. J. Bell, R. R. Bell, R. W. Alexander, and C. A. Ward, "Optical properties of the metals $\mathrm{Al}, \mathrm{Co}, \mathrm{Cu}, \mathrm{Au}, \mathrm{Fe}, \mathrm{Pb}, \mathrm{Ni}, \mathrm{Pd}, \mathrm{Pt}, \mathrm{Ag}, \mathrm{TI}$, and $\mathrm{W}$ in the infrared and far infrared," Applied Optics, vol. 22, no. 7, Avril 1983.

[28] A. Bajard, O. Aubreton, Y. Bokhabrine, B. Verney, G. Eren, A. Erçil, F. Truchetet, "3D Scanning of specular and diffuse metallic surfaces using an infrared technique", Optical Engineering 51, 06(2012) 0091-3286, 2012

[29] F. Marzani, Y. Voisin, L. F. C. Lew Yan Voon, and A. Diou, "Calibration of a threedimensional reconstruction system using a structured light source," Optical Engineering, vol. 41, no. 2, pp. 484-492, 2002. 